\title{
Photoproduction of $\omega$ mesons on nuclei near the production threshold
}

\author{
The CBELSA/TAPS Collaboration \\ M. Nanova ${ }^{1, a}$, J. Weil ${ }^{2}$, S. Friedrich ${ }^{1}$, V. Metag ${ }^{1}$, U. Mosel ${ }^{2}$, M. Thiel ${ }^{1}$, G. Anton ${ }^{3}$, J.C.S. Bacelar ${ }^{4}$, O. Bartholomy $^{5}$, \\ D. Bayadilov ${ }^{5,6}$, Y.A. Beloglazov ${ }^{6}$, R. Bogendörfer ${ }^{3}$, R. Castelijns ${ }^{4}$, V. Crede $^{5, b}$, H. Dutz ${ }^{7}$, A. Ehmanns $^{5}$, D. Elsner ${ }^{7}$, \\ K. Essig ${ }^{5}$, R. Ewald ${ }^{7}$, I. Fabry ${ }^{5}$, M. Fuchs ${ }^{5}$, Ch. Funke ${ }^{5}$, R. Gothe ${ }^{7, c}$, R. Gregor ${ }^{1}$, A.B. Gridnev ${ }^{6}$, E. Gutz $^{5}$, \\ S. Höffgen ${ }^{5}$, P. Hoffmeister ${ }^{5}$, I. Horn ${ }^{5}$, J. Hössl ${ }^{3}$, I. Jaegle ${ }^{8}$, J. Junkersfeld ${ }^{5}$, H. Kalinowsky ${ }^{5}$, Frank Klein ${ }^{7}$, \\ Friedrich Klein ${ }^{7}$, E. Klempt ${ }^{5}$, M. Konrad ${ }^{7}$, B. Kopf ${ }^{9,10}$, M. Kotulla ${ }^{1}$, B. Krusche ${ }^{8}$, J. Langheinrich ${ }^{7,10}$, H. Löhner ${ }^{4}$, \\ I.V. Lopatin 6 , J. Lotz ${ }^{5}$, S. Lugert ${ }^{1}$, D. Menze ${ }^{7}$, T. Mertens ${ }^{8}$, J.G. Messchendorp ${ }^{4}$, C. Morales ${ }^{7}$, R. Novotny ${ }^{1}$, \\ M. Ostrick ${ }^{7, d}$, L.M. Pant ${ }^{1, e}$, H. van Pee ${ }^{5}$, M. Pfeiffer ${ }^{1}$, A. Roy ${ }^{1, \mathrm{f}}$, A. Radkov ${ }^{6}$, S. Schadmand ${ }^{1, \mathrm{~g}}$, Ch. Schmidt $^{5}$, \\ H. Schmieden ${ }^{7}$, B. Schoch ${ }^{7}$, S. Shende ${ }^{4}$ h, G. Suft ${ }^{3}$, A. Süle ${ }^{7}$, V.V. Sumachev ${ }^{6}$, T. Szczepanek ${ }^{5}$, U. Thoma ${ }^{5}$, \\ D. Trnka ${ }^{1}$, R. Varma ${ }^{1, i}$, D. Walther ${ }^{5}$, Ch. Weinheimer ${ }^{5, j}$, and Ch. Wendel ${ }^{5}$ \\ 1 II. Physikalisches Institut, Universität Gießen, Germany \\ 2 Institut für Theoretische Physik I, Universität Gießen, Germany \\ 3 Physikalisches Institut, Universität Erlangen, Germany \\ 4 Kernfysisch Versneller Institut, Groningen, The Netherlands \\ ${ }^{5}$ Helmholtz-Institut für Strahlen- und Kernphysik Universität Bonn, Germany \\ 6 Petersburg Nuclear Physics Institute, Gatchina, Russia \\ 7 Physikalisches Institut, Universität Bonn, Germany \\ 8 Physikalisches Institut, Universität Basel, Switzerland \\ 9 Institut für Kern- und Teilchenphysik, TU Dresden, Germany \\ 10 Physikalisches Institut, Universität Bochum, Germany
}

Received: 24 August 2010 / Revised: 4 November 2010 Published online: 1 February 2011

(C) The Author(s) 2011. This article is published with open access at Springerlink.com Communicated by P. Braun-Munzinger

\begin{abstract}
The photoproduction of $\omega$ mesons on $\mathrm{LH}_{2}, \mathrm{C}$ and $\mathrm{Nb}$ has been measured for incident photon energies from 900 to $1300 \mathrm{MeV}$ using the CB/TAPS detector at ELSA. The $\omega$ lineshape does not show any significant difference between the $\mathrm{LH}_{2}$ and the $\mathrm{Nb}$ targets. The experiment was motivated by transport calculations that predicted a sensitivity of the $\omega$ lineshape to in-medium modifications near the production threshold on a free nucleon of $E_{\gamma}^{l a b}=1109 \mathrm{MeV}$. A comparison with recent calculations is given.
\end{abstract}

a e-mail: Mariana.Nanova@physik.uni-giessen.de

b Current address: Florida State University, Tallahassee, FL, USA.

Current address: University of South Carolina, Columbia, SC, USA.

d Current address: Physikalisches Institut, Universität Mainz, Germany.

e Current address: Nuclear Physics Division, BARC, Mumbai, India.

f Current address: IIT Indore, Indore, India.

g Current address: Forschunszentrum Jülich, Germany.

h Current address: Department of Physics, University of

Pune, Pune, India.

i Current address: Department of Physics, I.I.T. Powai, Mumbai, India.

j Current address: Universität Münster, Germany.

\section{Introduction}

Modifications of hadron properties in a strongly interacting environment have attracted a lot of attention and have been intensively studied both theoretically and experimentally. These studies were motivated by the expectation that chiral symmetry, a fundamental symmetry of Quantum Chromodynamics (QCD) in the limit of vanishing quark masses, may be restored in a nuclear medium at high temperatures or densities. In vacuum this symmetry is broken as visible in the low mass part of the hadronic spectrum: chiral partners - hadronic states with the same spin but opposite parity - like the $\rho$ and $a_{1}$ meson are different in mass while they should be mass degenerate if chiral symmetry were to hold. It turns out, however (for details see [1]) that a connection between chiral 
$E_{\gamma}=900-1100 \mathrm{MeV}$

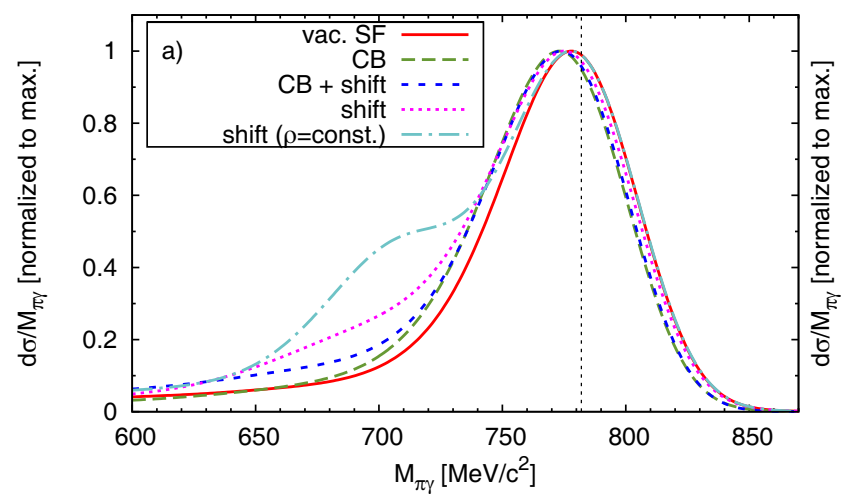

$\mathrm{E}_{\gamma}=900-1300 \mathrm{MeV}$

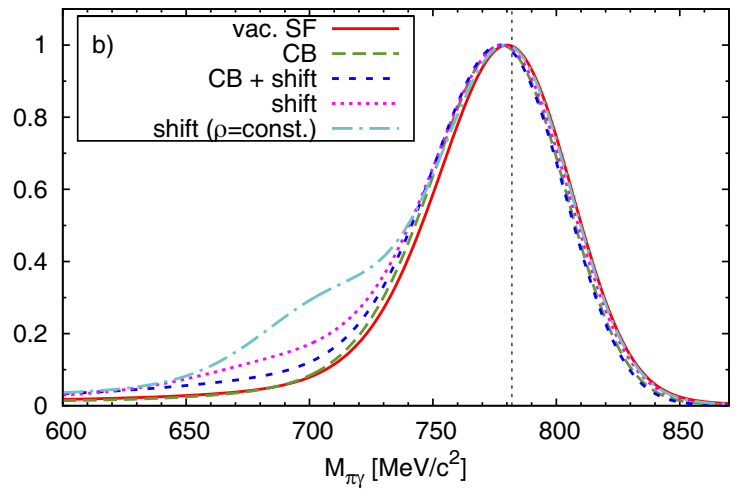

Fig. 1. (Color online) $\omega$ meson lineshape predicted for a $\mathrm{Nb}$ target in GiBUU transport model calculations for different inmedium modification scenarios: vacuum spectral function (solid line), collisional broadening of $\Gamma_{\text {coll }}=140 \mathrm{MeV}$ (long-dashed line), collisional broadening and an attractive mass shift of $-16 \%$ at nuclear matter density (short-dashed line) and mass shift without broadening (dotted line). The dash-dotted curve shows the results for a constant nuclear density of $\rho=0.6 \rho_{0}$. The signals are folded with the detector response given by eq. (1) with the parameters $\sigma=25 \mathrm{MeV}$ and $\tau=-0.09$ and take into account a $1 / E_{\gamma}$ weighting of the bremsstrahlung spectrum. a) Incident photon energies of 900-1100 MeV; b) incident photon energies of $900-1300 \mathrm{MeV}$.

symmetry restoration and hadronic in-medium spectral functions is much more involved. QCD sum rules provide a link between the quark-gluon sector and hadronic descriptions but do not fix the properties of hadrons in the strongly interacting medium. They only provide constraints for hadronic models which are still needed for calculating the in-medium self-energies of hadrons and their spectral functions.

Experimentally this field has been addressed in heavyion collisions and reactions with proton and photon beams. Light vector mesons are particularly suited for these investigations since - after production in a nuclear reaction - they decay in the nuclear medium with sizable probability because of their short lifetimes. Experimental results are summarized and critically evaluated in recent reviews $[1,2]$. Almost all experiments report a softening of the spectral functions of the light vector mesons $\rho, \omega$ and $\phi$. Increases in width are observed depending on the density and temperature of the hadronic environment. Mass shifts are only reported by the KEK group [3,4] who studied $\rho, \omega$ and $\phi$ production in proton nucleus reactions at $12 \mathrm{GeV}$. The claim of a mass shift of the $\omega$ meson in photoproduction on $\mathrm{Nb}[5]$ has not been confirmed in a reanalysis of the data [6].

In the latter experiment incident photon energies covered the range from $900-2200 \mathrm{MeV}$. Because of the increase of the production cross-section with photon energy most of the observed $\omega$ mesons are produced with photons of energy larger than $1500 \mathrm{MeV}$. For the energy range of $1500-2200 \mathrm{MeV}$ transport calculations $[7,8]$ have shown that the $\omega$ lineshape is rather insensitive to different inmedium modification scenarios like broadening or broadening and mass shift since most of the $\omega$ decays occur outside of the nuclear medium, even despite of a cut on the $\omega$ momentum $\left(p_{\omega} \leq 500 \mathrm{MeV} / c\right)$. Furthermore, due to the experimentally observed strong absorption of $\omega$ mesons in the nuclear medium [9] $\omega$ mesons produced in the interior of the nucleus are largely removed by inelastic reactions and do not reach the detector; information on possible in-medium mass shifts thereby gets lost. The limited sensitivity of the $\omega$ lineshape to in-medium modifications has been confirmed experimentally in [6].

Gallmeister et al. [7] argue that a search for medium effects would be much more promising for incident photon energies below or near the photoproduction threshold on a free nucleon of $E_{\gamma}^{l a b}=1109 \mathrm{MeV}$. New calculations along these lines illustrate in fig. 1 the expected sensitivity of the $\omega$ signal to various in-medium changes, such as mass shift with and without collisional broadening for two different energy ranges. It is seen that the lower-energy window indeed leads to a more pronounced - though not dramatic - sensitivity than the higher-energy one. This relatively weak sensitivity is to a large extent simply a consequence of the density profile of the nucleus that spans all densities from 0 to $\rho_{0}$ and thus smears any density-dependent signal. Assuming for the sake of the argument a density profile with a constant density of $0.6 \rho_{0}$ - roughly corresponding to the average density in nucleiand a sharp fall-off at the surface the dash-dotted line in fig. 1 is obtained; here the in-medium signal is significantly stronger. For a realistic nuclear density profile contributions to the spectral function from the surface dominate, suppressing contributions from higher-density regions [10].

In both energy windows a tail towards lower masses is predicted for the scenario of a dropping $\omega$ mass. This tail is due to $\omega$ mesons which are produced off-shell within the nucleus. In $[7,8]$ an even stronger enhancement in the low mass tail region was obtained. This calculation used a phenomenological method for the off-shell propagation while the present results are based on the theoretical framework provided by Leupold [11] and Juchem and Cassing [12] who have derived equations of motion for the testparticles that represent the spectral function in the transport simulation starting from the general Kadanoff-Baym 

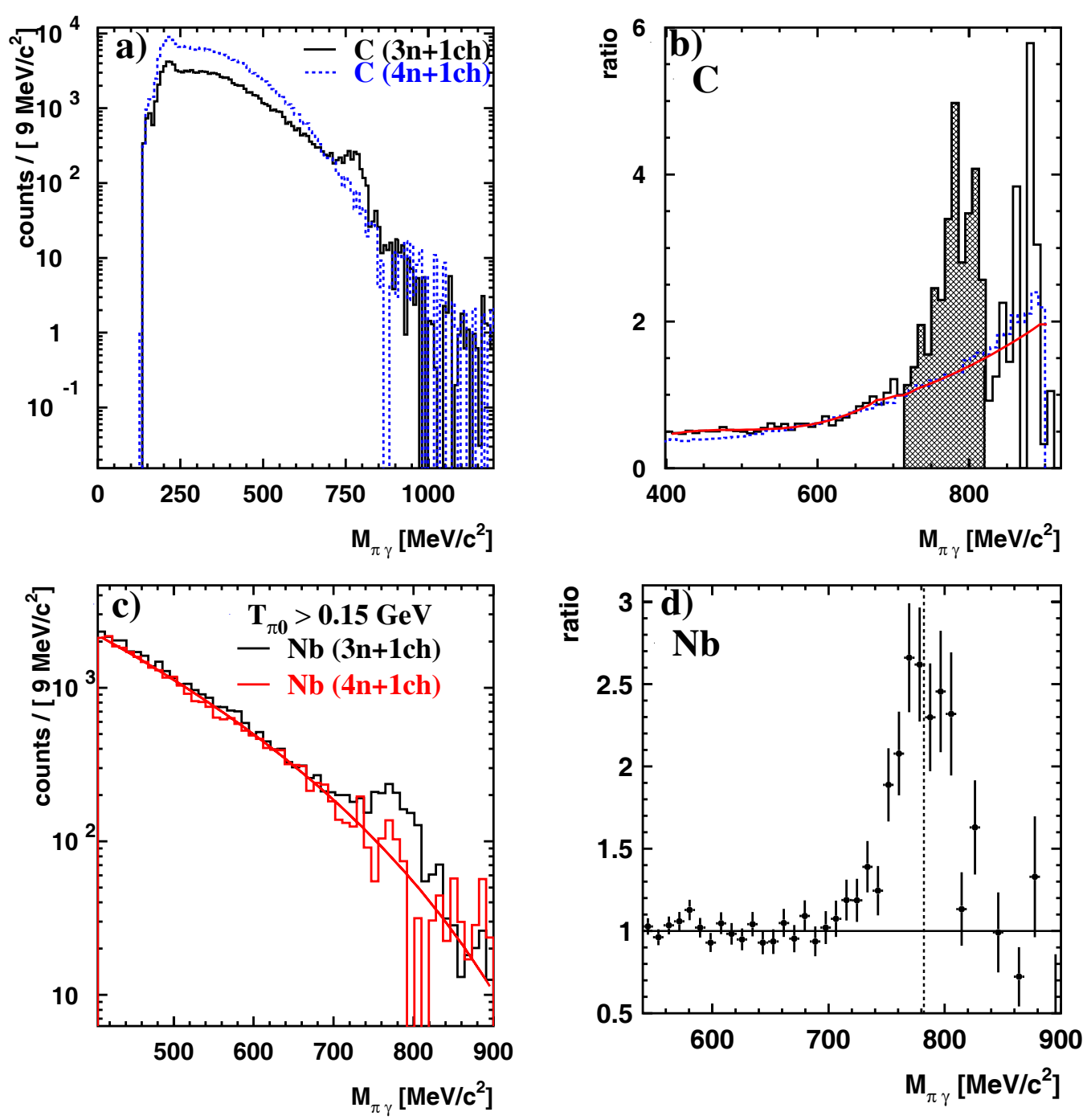

Fig. 2. (Color online) a) $\pi^{0} \gamma$ signal (solid curve) and background spectrum for the C target deduced from events with 4 neutral and 1 charged hit. b) Ratio of the distributions in a), reflecting the energy dependence of the probability for detecting 3 out of 4 photons relative to detecting all 4 photons per event. The red (solid) curve is a fit to the ratio ignoring the $\omega$ mass range. The blue (dashed) histogram represents a Monte Carlo simulation. c) The $\pi^{0} \gamma$ signal spectrum and the corrected and normalized background spectrum for the $\mathrm{Nb}$ target. The solid curve represents a fit to the background distribution. d) Ratio of the $\pi^{0} \gamma$ signal spectrum to the background spectrum for the Nb target generated from events with 4 neutral and 1 charged hit.

equations [13]. While the Leupold and Juchem-Cassing method has a firm theoretical basis it is nevertheless useful to remember that it relies on the assumption of adiabaticity, i.e. small gradients in space and time of all physical properties (potential, density, spectral function). Nonadiabatic effects such as those at level-crossings are not taken into account.

\section{Experimental set-up}

The experiment was performed at the ELSA electron accelerator facility $[14,15]$ at the University of Bonn, using the Crystal Barrel/TAPS detector set-up which provides an almost complete coverage of the full solid angle. The features of this calorimeter system and its capability for the detection of multi-photon final states have been de- scribed in detail elsewhere [16-18]. Tagged photon beams of $900-1300 \mathrm{MeV}$ were generated by bremsstrahlung and impinged in subsequent runs on $\mathrm{LH}_{2}, \mathrm{C}$ and $\mathrm{Nb}$ targets with thicknesses of $53 \mathrm{~mm}, 20 \mathrm{~mm}$ and $1 \mathrm{~mm}$ (30 mm diameter), respectively. The running conditions were the same as described in [6].

\section{Analysis}

The analysis of the data follows exactly the scheme described in detail in [6]. In fact, the data discussed here represent a subset of the data published in [6]. $\omega$ mesons were reconstructed in the reaction $\gamma A \rightarrow(A-1) p \omega \rightarrow$ $(A-1) p \pi^{0} \gamma \rightarrow(A-1) p \gamma \gamma \gamma$ from events with 3 photons and 1 proton in the final state. Figure $2 \mathrm{a}$ ) shows the $\pi^{0} \gamma$ 

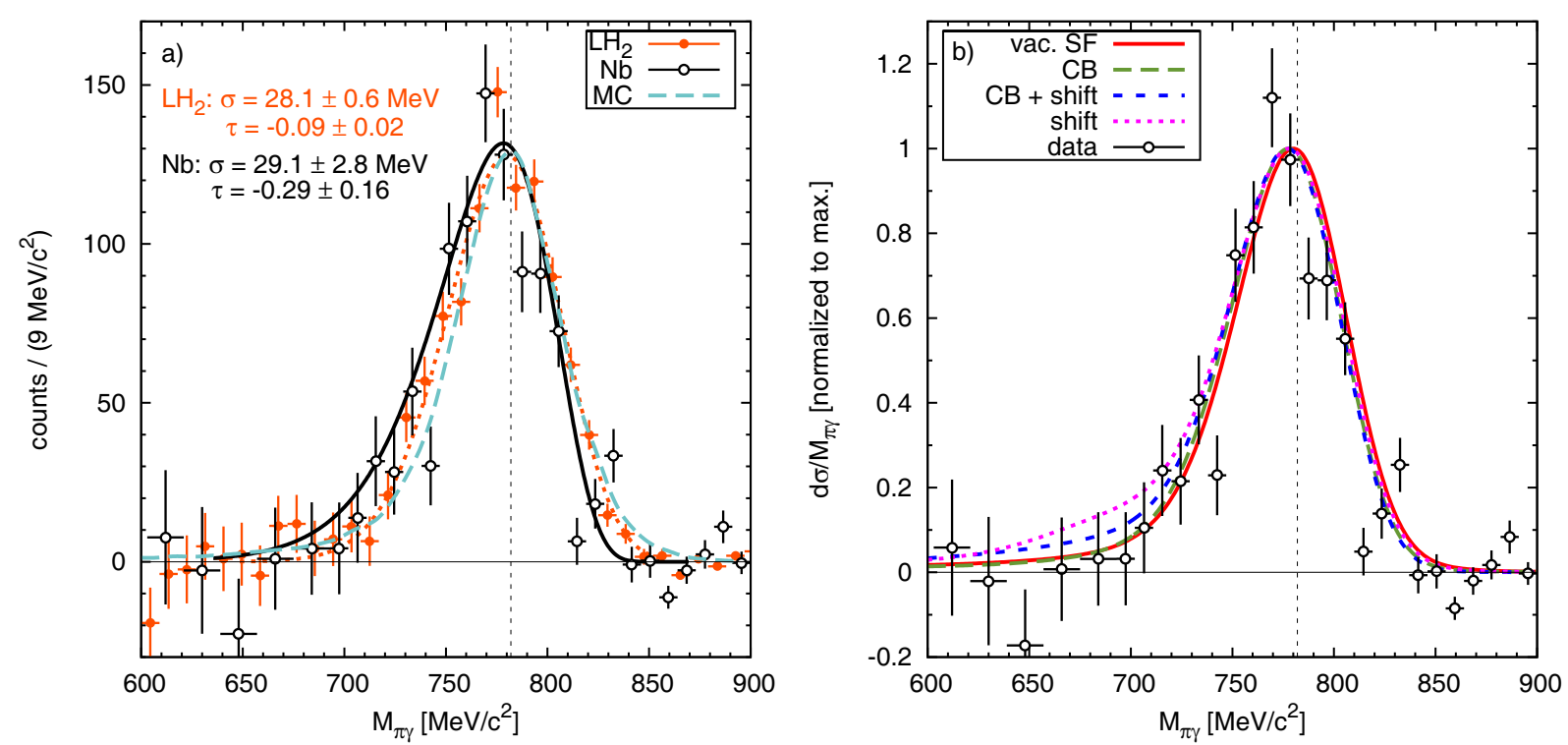

Fig. 3. (Color online) a) $\omega$ signal (solid points) for the Nb target ( $1 \mathrm{~mm}$ thick) and incident photon energies from $900-1300 \mathrm{MeV}$. The errors are purely statistical. Systematic errors introduced by the background subtraction are of the order of $5 \%$ (see fig. $2 \mathrm{~b}$ )). A fit curve to the data points (see text) is shown in comparison to the $\omega$ lineshape measured on a $53 \mathrm{~mm}$ long $\mathrm{LH}_{2}$ target and a Monte Carlo simulation; b) $\omega$ signal for the Nb target in comparison to recent GiBUU simulations for the following scenarios: no medium modification (solid line), in-medium broadening of $\Gamma_{\text {coll }}=140 \mathrm{MeV}$ at nuclear saturation density (long-dashed line), an additional mass shift by $-16 \%$ (short-dashed line) and mass shift without broadening (dotted line) The signals are folded with the detector response given by eq. (1) with the parameters $\sigma=25 \mathrm{MeV}$ and $\tau=-0.09$ and take into account a $1 / E_{\gamma}$ weighting of the bremsstrahlung spectrum.

invariant-mass spectrum for the carbon target for events with 1 charged and 3 neutral hits in the system. An $\omega$ signal is observed on a steeply falling background. As discussed in [6] this background stems dominantly from $\pi^{0} \pi^{0}$ and also $\pi^{0} \eta \rightarrow 4 \gamma$ final states where due to shower overlap in the detector or detector inefficiencies 1 out of the 4 photons escapes detection. Figure 2a) also shows the $\pi^{0} \gamma$ invariant-mass spectrum for events with 1 charged and 4 neutral hits where 1 neutral hit has been arbitrarily omitted to simulate the background in the 3 neutral and 1 charged spectrum. The slopes of the two spectra are different, reflecting the energy dependence of the probability to register only 3 out of 4 photons relative to detecting all 4 photons. The ratio of the two spectra in fig. 2a) is given in fig. 2b). This ratio is a smooth function of the invariant mass as verified by Monte Carlo simulations, also shown in fig. 2b). Since no strong in-medium effects are expected for a light nucleus like carbon this ratio can be applied to correct the $\pi^{0} \gamma$ background spectrum derived from events with 1 charged and 4 neutral hits measured for the $\mathrm{Nb}$ target. Figure 2c) shows the $\pi^{0} \gamma$ invariant-mass spectrum for $\mathrm{Nb}$ together with this background distribution after applying this correction. The normalization of the background spectrum is done by requesting the same number of events in the mass range from $400-900 \mathrm{MeV}$, excluding the counts in the $\omega$ peak which account for only $2 \%$ of the total yield in this mass range. It is important to note that this determination of the background in magnitude and shape does not pay any attention to the $\omega$ signal region. The background in the ( 3 neutral +1 charged $)$ signal spectrum is well reproduced by the corrected ( 4 neutral + 1 charged) spectrum. This is demonstrated by the ratio of the two spectra shown in fig. $2 \mathrm{~d}$ ). In the mass range from $400-700 \mathrm{MeV}$ the average deviation from unity is $5 \%$. This uncertainty determines the systematic error in the background subtraction.

\section{Comparison to the $\mathrm{LH}_{2}$ reference measurement and to GiBUU simulations}

After subtraction of the background spectrum from the $\pi^{0} \gamma$ signal spectrum the $\omega$ lineshape shown in fig. 3a) is obtained for the Nb target. The experimental distribution has been fitted using the Novosibirsk function [19]:

$$
f(x)=A \cdot \exp \left[-\frac{1}{2}\left(\frac{\ln q_{x}}{\tau}\right)^{2}+\tau^{2}\right],
$$

where

$$
q_{x}=1+\frac{\left(x-x_{0}\right)}{\sigma} \cdot \frac{\sinh (\tau \sqrt{\ln 4})}{\sqrt{\ln 4}} .
$$

Here $A$ is the amplitude of the signal, $x_{0}$ is the peak position, $\sigma$ is FWHM/2.35 and $\tau$ is the asymmetry parameter. This function takes into account the tail in the region of lower invariant masses resulting from the energy response of the calorimeters. The fit in the mass range 700 to $820 \mathrm{MeV}$ is compared with the $\omega$ signal measured on the $\mathrm{LH}_{2}$ target and with a Monte Carlo simulation of the $\omega$ 
signal in fig. 3a). It should be noted that the $53 \mathrm{~mm}$ length of the $\mathrm{LH}_{2}$ target leads to an increase in the $\omega$-signal width by less than $10 \%$. The agreement between the $\omega$ signal on the $\mathrm{LH}_{2}$ target with the Monte Carlo simulation demonstrates that the detector response is under control. The fit parameters are $\sigma=(29.1 \pm 2.8) \mathrm{MeV}, \tau=-0.29 \pm 0.16$ for $\mathrm{Nb}$ and $\sigma=(28.1 \pm 0.6) \mathrm{MeV}, \tau=-0.09 \pm 0.02$ for $\mathrm{LH}_{2}$, respectively. The fit of the data with the function of eqs. (1), (2) yields a $\chi^{2} / \mathrm{DoF}=18.95 / 12$ with a $\chi^{2}$ probability of $9.0 \%$ while a fit of the data with the $\omega$ lineshape measured on the $\mathrm{LH}_{2}$ target gives a $\chi^{2} / \mathrm{DoF}=32.8 / 15$, corresponding to a $\chi^{2}$ probability of $0.5 \%$. Nevertheless, in view of the systematic and statistical uncertainties no significant deviation from the reference signals is claimed. Higher statistics will be needed to draw any conclusion. Corresponding data have been taken at MAMI$\mathrm{C}$ using the Crystal Ball/TAPS set-up. The analysis is ongoing.

In fig. $3 \mathrm{~b}$ ) the measured $\omega$ signal is compared to predictions of transport calculations using the GiBUU model [20] for the same scenarios as in fig. 1. While all the curves seem to underestimate the data slightly on the low mass side of the $\omega$ peak, the experimental data obviously do not allow to distinguish between the various theoretical scenarios, in contrast to initial expectations.

\section{Conclusions}

The photoproduction of $\omega$ mesons on $\mathrm{LH}_{2}, \mathrm{C}$ and $\mathrm{Nb}$ targets has been measured for incident photon energies from $900-1300 \mathrm{MeV}$, i.e. near the photoproduction threshold on a free nucleon of $E_{\gamma}^{l a b}=1109 \mathrm{MeV}$. The experimentally observed $\omega$ signal does not allow to distinguish between various in-medium scenarios which - also near threshold - lead all to only a weak tail at low invariant masses. Access to the in-medium spectral function of vector mesons is thus very limited, mainly due to the dependence of the in-medium properties such as mass and width on the nuclear density $[10,21]$ and the inherent density smearing caused by the density profile of nuclei. On the other hand, transparency measurements [22,23] can give at least access to the imaginary part of the in-medium self-energy of the hadron. Another promising tool could be the measurement of excitation functions [24]. Such experiments are presently being analyzed.
We thank the scientific and technical staff at ELSA and the collaborating institutions for their important contribution to the success of the experiment. We gratefully acknowledge detailed discussions with S. Leupold and P. Mühlich. This work was supported financially by the Deutsche Forschungs Gemeinschaft through SFB/TR16, by the Schweizerischer Nationalfond and by the Stichting voor Fundamenteel Onderzoek der Materie (FOM) and the Nederlandse Organisatie voor Wetenschappelijk Onderzoek (NWO).

Open Access This article is distributed under the terms of the Creative Commons Attribution Noncommercial License which permits any noncommercial use, distribution, and reproduction in any medium, provided the original author(s) and source are credited.

\section{References}

1. S. Leupold, V. Metag, U. Mosel, Int. J. Mod. Phys. 19, 147 (2010) (arXiv:0907.2388 [nucl-th]).

2. R. Hayano, T. Hatsuda, arXiv:0812.1702 (2008).

3. M. Naruki et al., Phys. Rev. Lett. 96, 092301 (2006).

4. R. Muto et al., Phys. Rev. Lett. 98, 042501 (2007).

5. D. Trnka et al., Phys. Rev. Lett. 94, 192303 (2005).

6. M. Nanova et al., Phys. Rev. C 82, 035209 (2010) (arXiv:1005.5694 [nucl-ex]).

7. K. Gallmeister, M. Kaskulov, U. Mosel, P. Mühlich, Prog. Part. Nucl. Phys. 61, 283 (2008).

8. P. Mühlich, PhD Thesis, University of Giessen (2006).

9. M. Kotulla et al., Phys. Rev. Lett. 100, 192302 (2008).

10. U. Mosel, S. Leupold, V. Metag, arXiv:1006.5822 [nucl-th].

11. S. Leupold, Nucl. Phys. A 672, 475 (2000).

12. W. Cassing, S. Juchem, Nucl. Phys. A 677, 445 (2000).

13. L.P. Kadanoff, G. Baym, Quantum Statistical Mechanics (Benjamin, New York, 1962).

14. D. Husmann, W.J. Schwille, Phys. Bl. 44, 40 (1988).

15. W. Hillert, Eur. Phys. J. A 28, 139 (2006).

16. E. Aker et al., Nucl. Instrum. Methods A 321, 69 (1992).

17. R. Novotny et al., IEEE Trans. Nucl. Sci. 38, 392 (1991).

18. A.R. Gabler et al., Nucl. Instrum. Methods A 346, 168 (1994).

19. B. Aubert et al., Phys. Rev. D 70, 112006 (2004).

20. http://gibuu.physik.uni-giessen.de.

21. J. Lehr, U. Mosel, Phys. Rev. C 64, 042202 (2001). (arXiv:nucl-th/0105054).

22. M. Kaskulov, E. Hernandez, E. Oset, Eur. Phys. J. A 31, 245 (2007).

23. P. Mühlich, U. Mosel, Nucl. Phys. A 773, 156 (2006).

24. P. Mühlich, T. Falter, U. Mosel, Eur. Phys. J. A 20, 499 (2004) (arXiv:nucl-th/0310067). 